\title{
Issues of incompatibility of dipole beam separation schemes with EIC physics
}

\author{
E. Aschenauer, A. Kiselev, B. Parker, R. Petti
}

\section{Collider-Accelerator Department Brookhaven National Laboratory Upton, NY 11973}

\author{
U.S. Department of Energy \\ Office of Science, Office of Nuclear Physics
}

Notice: This document has been authorized by employees of Brookhaven Science Associates, LLC under Contract No. DE-SC0012704 with the U.S. Department of Energy. The United States Government retains a nonexclusive, paid-up, irrevocable, world-wide license to publish or reproduce the published form of this document, or allow others to do so, for United States Government purposes. 


\section{Issues of Incompatibility of Dipole Beam Separation Schemes with EIC Physics}

Elke-C. Aschenauer, Alexander Kiselev, Brett Parker, and Richard Petti 


\section{Introduction}

This report addresses the question can the risk of an eRHIC machine be reduced by replacing the crab-cavities through a dipole field in the IR to separate the electron and hadron beams. In the following several scenarios to integrate a separation dipole in the IR design will be described. For each of the scenarios the challenges and problems to realize this in practice will be detailed. In addition the impact on the detector design and the EIC physics program will also be discussed.

Several physics requirements need to be integrated into the interaction region design, in order to perform the physics program as described in the EIC White Paper [2] and in the RHIC Design Study [2]. These requirements are also described in detail on a dedicated page of the eRHIC-wiki [1]. The main requirements are described here.

\subsection{Requirements in the outgoing hadron beam direction}

To veto the breakup of nuclei in the reaction e $+A \rightarrow e^{\prime}+A^{\prime}+V M$ it is critical to have a large acceptance for breakup neutrons at zero degrees. Simulations show that a $+/-4$ mrad cone needs to be passed undisturbed to the Zero Degree Calorimeter (ZDC) in order to detect these breakup neutrons efficiently.

To ensure exclusivity in deeply virtual Compton scattering (DVCS) reactions $e^{+} p \rightarrow e^{\prime}+p^{\prime}+\gamma$ it is critical to detect the forward going scattered proton, and to measure its transverse momentum very accurately. The scattered proton momentum versus its scattering angle in the laboratory frame is shown in Figure 1. A common technique to detect these protons is to install Roman Pots symmetrically around the beam in the warm sections of the outgoing proton beam. A distance of $10 \sigma$ from the Roman Pot to the core of the beam is required for safety reasons. The physics requirement is to detect protons with the smallest and largest possible scattering angles. This translates to a requirement on the $10 \sigma$ of proton beam divergence of less than $0.68 \mathrm{mrad}$ at $250 \mathrm{GeV}$, and $1.7 \mathrm{mrad}$ at $100 \mathrm{GeV}$. The maximum angle that needs to be transported through the system is $5 \mathrm{mrad}$ at $250 \mathrm{GeV}$ and $13 \mathrm{mrad}$ at $100 \mathrm{GeV}$. Note that the main detector acceptance starts only at $\sim 30 \mathrm{mrad}$.

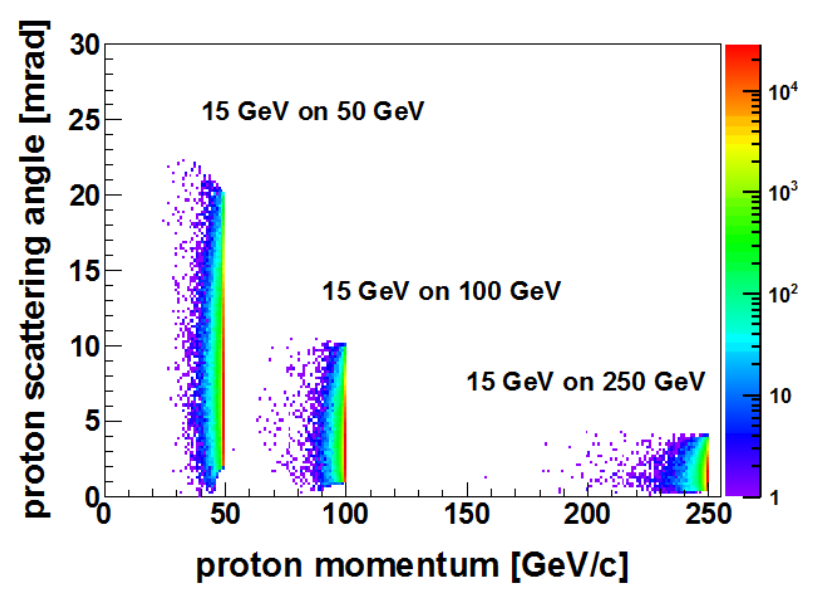

Figure 1: The scattered proton momentum vs. scattering angle in the laboratory frame for DVCS events with different beam energy combinations. The following cuts have been applied: $1 \mathrm{GeV}^{2}<Q^{2}<100$ $\mathrm{GeV}^{2}, 0.01<y<0.85,10^{-5}<x<0.5$ and $0.01<t<1$ $\mathrm{GeV}^{2}$. The angle of the recoiling hadronic system is directly and inversely correlated with the proton energy, therefore it decreases with increasing proton energy.

\subsection{Requirements in the outgoing lepton beam direction}

To match the high statistical precision that an EIC will achieve for basically all key measurements, it is extremely important to reduce systematic uncertainties. Most critical is the bunchby-bunch measurement of luminosity using bremsstrahlung events e+p/A $\rightarrow$ e'+p'/A'+ $\gamma$. The requirements are to know the absolute luminosity to better than $1 \%$ and to determine the relative luminosity to $10^{-5}$. Simulations of this fully calculable process show that the natural angular distribution of the bremsstrahlung photons is contained in a cone of 0.05 mrad. The width of the 
bremsstrahlung photon cone that is to be transported through the interaction region at zero degrees is purely given by the electron beam divergence.

It is critical to detect the scattered lepton below a $\mathrm{Q}^{2}$ of $0.1 \mathrm{GeV}^{2}$, the limit seen in the main detector. In order to do so it is necessary to install a special electromagnetic calorimeter to detect leptons scattered with very small scattering angles. This calorimeter needs to be installed after a bend of the lepton beam, so that the scattered leptons are separated from the main core of the outgoing electron beam. It is critical to suppress synchrotron radiation hitting the calorimeter as much as possible. Leptons with $\mathrm{Q}^{2}$ values as small as $10^{-4}$ can be detected in the nominal linac-ring design.

\section{IR design with a dipole}

The eA physics program comprises several measurements that have unique requirements that make a qualitatively different impact on the IR design. For eA it is not enough to simply separate the electron and hadron beams; we must also cleanly separate the forward hadron beam from neutral particles coming from the IP. This is inherently a two-stage process. Although we can use quite strong fields focusing the hadrons and separating them from the neutrals, the electron beam would produce unacceptably large synchrotron radiation (synrad) in a strong magnetic field and therefore must first be put into an independent low-field aperture. The dominant forward aperture requirement, that of accepting a 5 mrad neutron cone from the IP, is substantially larger than those for the circulating hadron beam. But requiring oversized hadron beam apertures brings with it two quite negative impacts on the forward IR magnet designs. First for a given peak field strength, a larger hadron aperture in a quadrupole magnet means that the magnet gradient is lower and focusing is weaker. This impact is further compounded in that with weaker focusing the quadrupole length has to be increased, so the aperture has to increase further to accommodate the neutron cone and this can lead to a runaway situation where there is no good optics solution available that keeps beta peaks down to stay within accelerator chromaticity design limits. The second impact of overly large hadron apertures applies both for quadrupoles and dipoles in that the combination of a large peak coil fields and large coil radii increases the magnitude of external fields. A significant increase in the external field makes shielding the electron beam from these fields go from being challenging to unfeasible.

Taking the above into account we find that for an IR layout to satisfy the eA neutron cone acceptance requirement even a small increase in the longitudinal space required for the initial electron/hadron beam separation can propagate through the IR design to have substantial beam optics impacts. This is the main reason why the baseline eA IR design beam separation scheme employs a crossing angle. A crossing angle scheme is inherently more efficient than a separation dipole scheme because a separation dipole requires longitudinal distance to reach its full deflection angle but with a crossing angle the beams separate immediately on either side of the IP. Also unless the separation dipole is either beyond the detector boundary or integrated with the detector solenoid, here denoted a Detector Integrated Dipole (DID), the separation dipole will block a significant fraction of the forward physics acceptance. A large aperture dipole has an even larger outer magnetic structure and it is this structure will block other experimental physics acceptance outside the $5 \mathrm{mrad}$ neutron cone. Note this is also why it is not feasible to put quadrupole focusing inside the eA detector region; the magnet structure for such a quadrupole would also take away a region of forward acceptance beyond 5 mrad. Overlaying the detector solenoidal field with extra non-solenoidal field components also has significant impact on the subdetector technology choices and detector magnet optimization which will be addressed in a later section.

For eRHIC eA physics IR layout any decision to use a separation dipole also brings with it a subtle design conflict, that is absent in a crossing angle scheme, between the forward and rear sides due to the production of synrad. For eA the large forward hadron magnet apertures pretty much require 
superconducting coil technology for reasonable hadron/neutral beam separation and focusing and unless we are willing to have drastically different hadron optics and beam geometry layout differences between the forward and rear sides, we end up needing to use superconducting magnets for the hadron beam on the rear side too. But the separation dipoles create a powerful synrad fan that sweeps back and forth between the electron and hadron beam axes from which these superconducting IR magnets must be protected. For HERA-II the main hadron IR magnet apertures set by the circulating beam size and the first few hadron magnets were normal conducting, warm. These warm magnets had vertically split coil structures that allowed the majority of the synrad generated by the dipole separation scheme to cleanly pass on to absorbers far from the IP. Even so the small amount of secondary albedo backscatter coming back at small angles from the remaining fraction of the synrad which hit a small septum protection absorber created enough background in inner detector components to be problematic and a definite limiting factor for the initial HERA-II physics running. While these initial background issues were eventually mitigated through a combination improvements in the anti-backscatter masking scheme and other hardware changes, HERA-II experiments were still fortunate in not having to deal with large angle synrad albedo backscatter. As we shall see later, due to purely geometrical considerations, synrad backscatter that comes back at large angles is quite a bit harder to protect against. Unfortunately it is just not practical to split superconducting quadrupole magnets in the same way and making extended field free slots in saturated superconducting magnet yokes is both magnetically and mechanically unrealistic.

In order to illustrate the preceding arguments, we will investigate a few representative scenarios for a range of separation dipole assumptions. First consider the case shown in Figure 2 where the separation dipoles are kept completely outside the detector and the synrad critical energy for the eRHIC $20 \mathrm{GeV}$ electron beam energy is close to the $110 \mathrm{keV}$ value of HERA-II. Note that even with 25 mrad deflection (2.5 times the $10 \mathrm{mrad}$ linac-ring baseline angle), the first superconducting hadron/electron IR magnets in the forward direction must be placed well beyond $10 \mathrm{~m}$ from the IP $\left(4.5 \mathrm{~m} \mathrm{~L}^{*}\right.$ for linacring baseline) because otherwise there is not enough room between the e-beam and the forward neutron cone for magnet structure. This occurs because for the separation dipole bend center is $6.5 \mathrm{~m}$ from the IP, where the neutron cone is already quite extended, and with 20 mrad angle there is barely enough lever arm for the electron beam to be outside the expanding neutron cone at $10 \mathrm{~m}$.

On the rear side we find a synrad fan that started with the beginning of the separation dipole at the $8.5 \mathrm{~m}$ on the forward side that is a very wide inside the detector and beyond. In particular at $10 \mathrm{~m}$ on the rear side, the synrad fan extends an additional $325 \mathrm{~mm}$ beyond the e-beam; it is quite impractical to pass such a wide band through a the downstream rear side superconducting magnet structure. Also note that the forward separation dipole itself needs a large, $85 \mathrm{~mm}$, clear vertical aperture to pass the neutron cone. For our horizontally bending beam separation dipole, increasing the vertical aperture costs space for more amp-turns of coil and yoke which leads to a large magnet body. If we were to try to reduce the width of the synrad fan by pushing this dipole a little bit inside the $4.5 \mathrm{~m}$ detector boundary, this large magnet structure would be in the way of a large fraction of the forward physics acceptance beyond 5 mrad.

We could look to mitigate some of the loss of physics acceptance from having separation dipoles inside the detector by integrating the dipole coils with the detector solenoid, e.g. the DID scenario shown in Figure 3. Using a DID avoids the issue of blocking forward/rear acceptance but at the cost of complicating the detector magnet design. Since the beam separation starts much closer to the IP, the geometry is much closer to that which could be achieved using a crossing angle geometry. Still for equal bend and crossing angles, the separation that is achieved between the incoming beam and the edge of the forward neutron cone is less with a separation dipole scheme. While the edge of the synrad band on the rear side now is very much closer to the e-beam than for scenario 1, there is still a fairly wide synrad fan that extends all the way down to the axis of the hadron bean. The superconducting IR magnets have to be protected from this synrad fan. Unfortunately the DID coil 
geometry implied by integrating a pair of large diameter dipole coils with the baseline eRHIC detector solenoid leads to coils that are very inefficient at producing separation field.

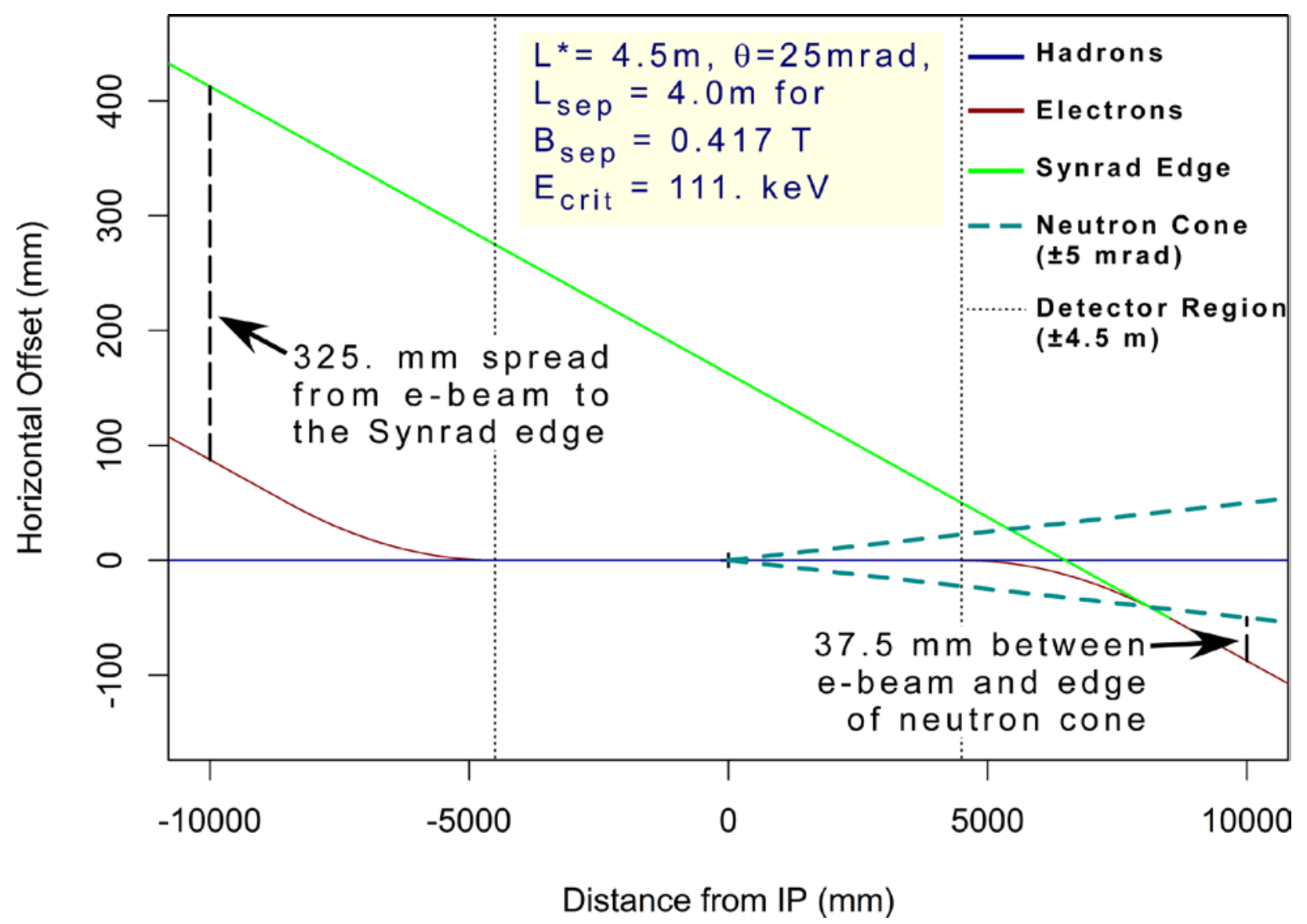

Figure 2: Scenario 1 with the separation dipoles completely outside the detector and synrad critical energy at $20 \mathrm{GeV}$ similar to HERA-II. Note that even with 25 mrad deflection the first hadron/electron IR magnet in the forward direction can only be beyond $10 \mathrm{~m}$ from the IP because otherwise there is not enough separation between the e-beam and the forward neutron cone for the magnet structure. Since the separation bend starts at $8.5 \mathrm{~m}$ there is a very wide synrad fan both inside the detector and beyond. In particular at $10 \mathrm{~m}$ on the rear side the synrad fan extends $325 \mathrm{~mm}$ beyond the e-beam and it is not practical to pass this wide band through a superconducting magnet structure. Finally the forward separation dipole itself needs large, $85 \mathrm{~mm}$, clear vertical aperture to pass the neutron cone and would block a large fraction of the forward acceptance outside $5 \mathrm{mrad}$ if it were to be pushed inside the $4.5 \mathrm{~m}$ detector boundary. 


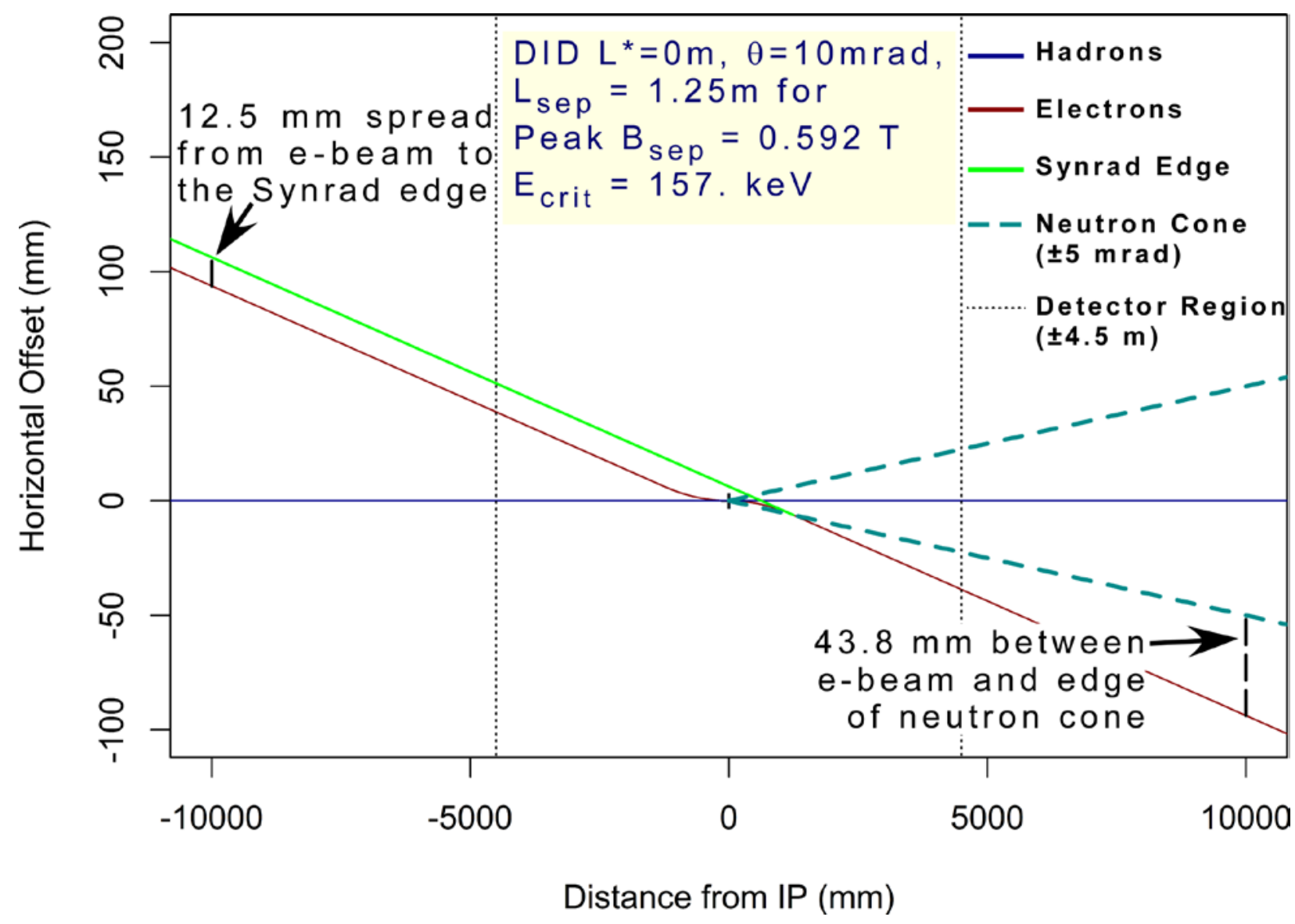

Figure 3: Scenario 2 with the separation dipole coils integrated with the detector (DID). Integrating the dipole coils with the detector solenoid avoids the issue of blocking forward/rear acceptance but at the cost of complicating the detector magnet design. Now we find that with beam separation starting much closer to the IP, the geometry is closer to that which could be achieved using a crossing angle geometry. But for the same crossing angle, the separation between the incoming beam and the edge of the forward neutron cone is reduced. While the edge of the synrad band on the rear side now is much closer to the e-beam than for scenario 1, there is still a wide synrad fan that extends down to the axis of the hadron bean. The superconducting IR magnets have to be protected from this synrad fan. Unfortunately the DID coil geometry implied by integrating a two $1.25 \mathrm{~m}$ long dipole coils with a $1 \mathrm{~m}$ radius detector solenoid coil, antisymmetrically about the IP, leads to coils that are very inefficient at producing separation field and as such are just not practical.

The baseline eRHIC detector has a $1 \mathrm{~m}$ solenoid coil radius and $2.5 \mathrm{~m}$ length. So we place two DID coils with radii greater than $1 \mathrm{~m}$, that are each $1.25 \mathrm{~m}$ long, in an anti-symmetric arrangement about the IP to produce the field profile shown in Figure 4. In Figure 4 we plot the separation field at the detector axis as a function of distance to the IP on the forward side of the detector. On the rear side (not plotted) the field direction is reversed and by symmetry the field is zero at the IP. The DID coils are so short relative to their coil radii that they are "mostly coil ends" and produce a longitudinally varying field on axis. The peak field seen by the e-beam is about $10 \%$ higher than that for an equivalent $1.25 \mathrm{~m}$ uniform field and this leads to an increase of the synrad critical energy. There is also a long tail that extends beyond $1.25 \mathrm{~m}$. The presence of this extra, non-uniform field in the central detector region is problematic for operation of the baseline TPC experimental detector technology and the long range field would have bearing on use of a RHIC detector as will be explained in later sections. 


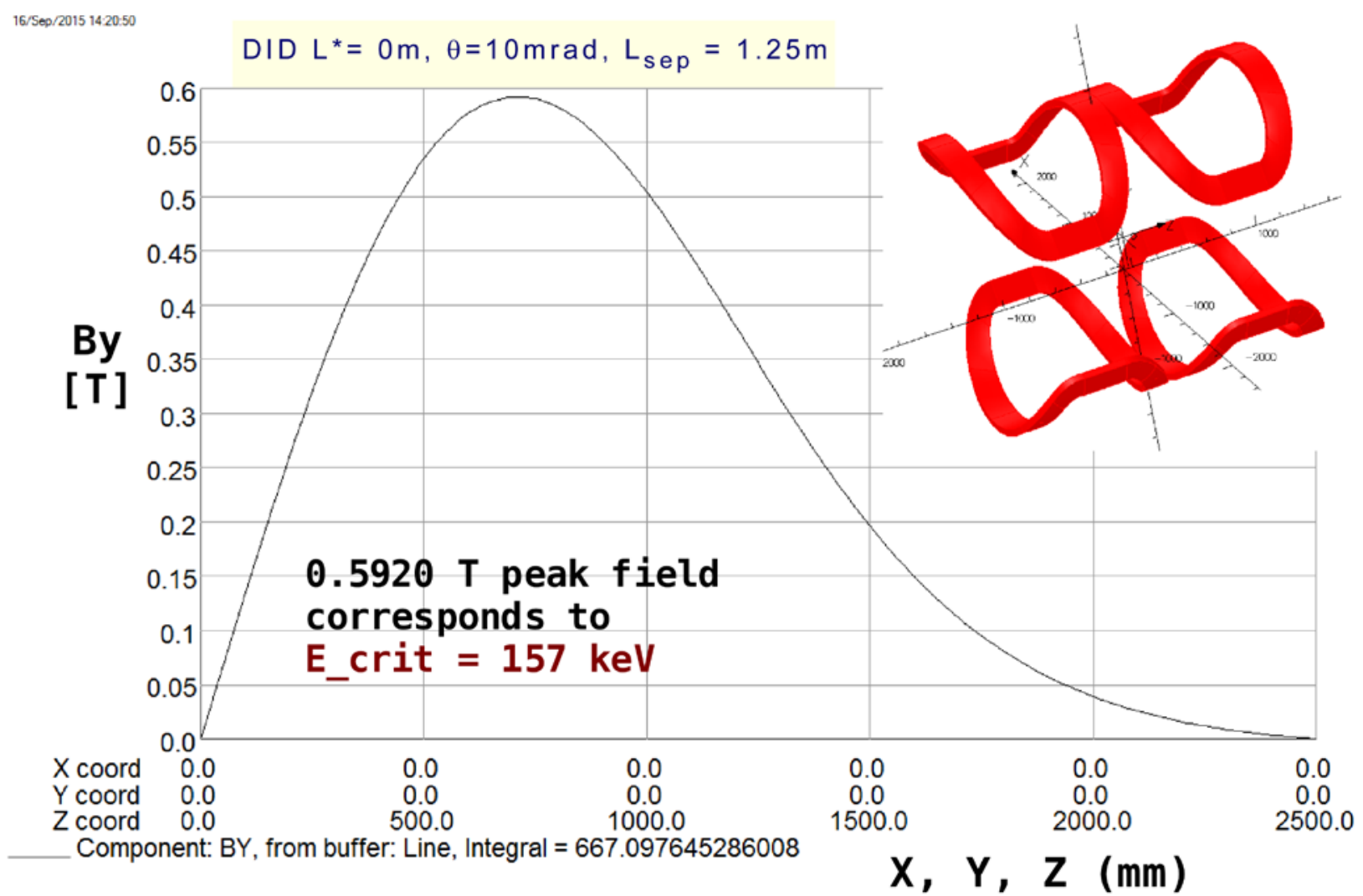

Figure 4: Plot of the separation field at the detector axis as a function of distance to the IP on the forward side of the detector. On the rear side the field is reversed and by symmetry the field is zero at the IP. The peak field seen by the e-beam is about $10 \%$ higher than the average value and this leads to an increase of the synrad critical energy. The presence of this extra, non-uniform field in the central detector region is problematic for operation of the TPC-based tracker.

These DID coils would certainly have a major impact on the performance of the solenoid coil itself as can be seen from the plot in Figure 5 of the separation field at the DID coil with the detector solenoid turned off. Due to the very poor length/diameter aspect ratio of these coils, this coil geometry is not very efficient at creating field on axis and since the coils on either half of the detector have opposite polarity they tend to cancel each other over a broad region. The result is that only about $10 \%$ of the field found at the coil surface is actually useful for deflecting the e-beam. But in fact field from the DID coil at the solenoid coil is already greater in magnitude than that produced by the detector solenoid itself. So the detector solenoid design and operation would be very strongly impacted by these DID coils. A particularly simplified coil structure was chosen for presentation in these figures for illustrative purposes; however, even using a more complex, optimized coil structure, the conclusion remains that a DID coil which closely matches the baseline e-RHIC detector solenoid geometry would be very inefficient and presents a huge detector design perturbation. For a DID coil beam separation to work we are forced to consider major modifications of the baseline detector concept, both in detection technology and the solenoid design. 


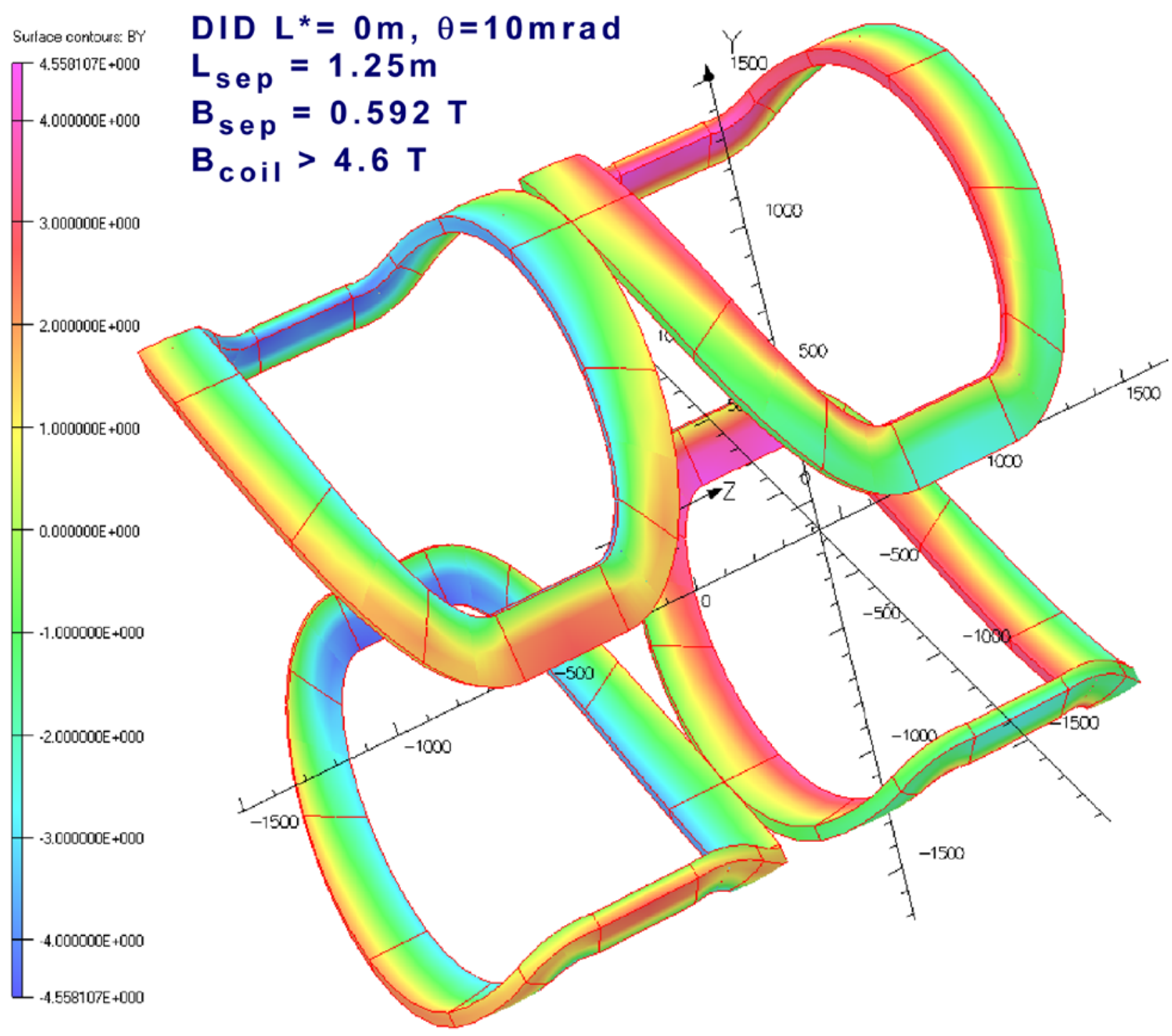

Figure 5: Plot of the separation field at the DID coil itself. Due to the very poor length/diameter aspect ratio of these coils this coil geometry is very inefficient at creating field on axis. Also the coils on either half of the detector have opposite polarity and therefore tend to cancel each other. Thus only slightly greater than $10 \%$ of the field at the coil surface is actually useful for deflecting the e-beam.

The detector proposed for the $\mathrm{LHeC}$ provides an example where the detector geometry is adjusted to be more favorable for DID operation. The LHeC magnet layout and central field profile are shown in Figure 6. Note that the LHeC DID superconducting coil aspect ratio is significantly stretched with respect to the baseline eRHIC detector having a smaller diameter while being much longer to the point that the combined LHeC DID beam separation dipoles extend beyond the central solenoid region. For the LHeC beam separation scheme there are additional small aperture warm dipoles on both sides of the central detector region to complete the full $\pm 9 \mathrm{~m}$ of separation bending. The vertical aperture of the extra warm dipole section on the forward side of the central detector would not be adequate to pass the forward neutron cone needed to do eRHIC eA physics. The field plot in Figure 6 demonstrates the advantage of having a more favorable DID length/diameter ratio in that now the dipole separation component, $\mathrm{By}$, at the detector axis is much flatter and with less cancellation between the two opposite polarity DID coils. With this more favorable DID geometry the LHeC DID produces $0.3 \mathrm{~T}$ separation field at the electron beam while keeping the total field at the detector solenoid coil below $4 \mathrm{~T}$. 

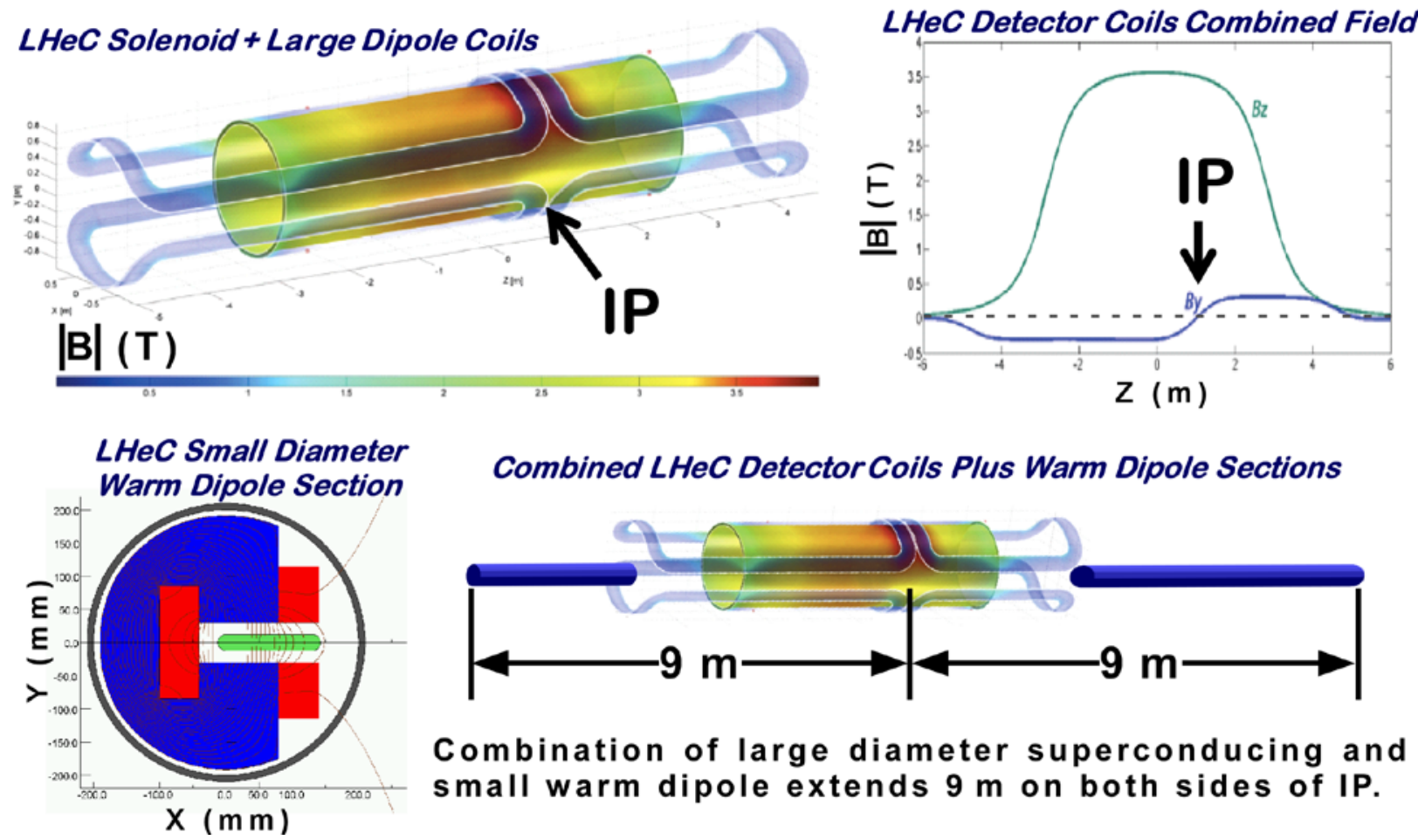

Combined LHeC Detector Coils Plus Warm Dipole Sections

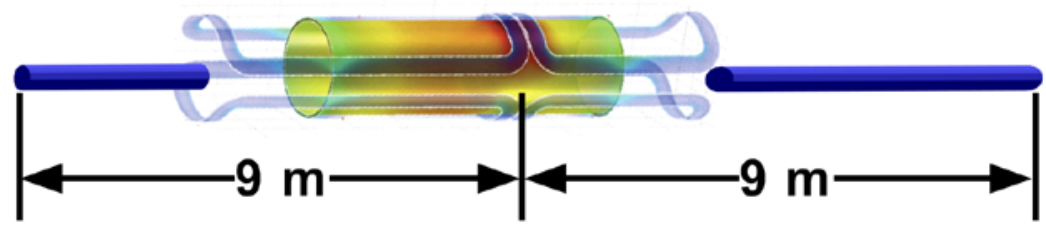

Combination of large diameter superconducing and small warm dipole extends $9 \mathrm{~m}$ on both sides of IP.

Figure 6: LHeC Separation Dipole Scheme. The detector proposed for the LHeC provides an example where the detector coil geometry is adjusted to be more favorable for DID operation. Note that the superconducting coil aspect ratio is significantly stretched with respect to the baseline eRHIC detector and has a much smaller diameter while being much longer. The LHeC beam separation scheme has additional small aperture warm dipoles on both sides of the central detector region to complete the separation bend and is therefore not compatible with passing the forward neutron cone as required for the eRHIC eA nuclear physics program. Note that unlike the convention for the other figures in this note, the hadron beam direction is reversed, going right to left, so the forward physics region is on the left and the rear side is on the right.

In Figure 7 we show the geometry for an appropriately modified eRHIC detector. With respect to the eRHIC baseline geometry the DID coil length is doubled while the coil radius is halved so that the new geometry looks more like the LHeC configuration. Unlike the LHeC configuration we do not use additional warm separation dipole sections outside the detector because we must preserve eA forward/rear acceptance. With $20 \mathrm{mrad}$ e-beam deflection angle there is now adequate space at $10 \mathrm{~m}$ in the forward direction for a superconducting coil structure to start focusing the hadron beam and to start separating the forward charged particles from the neutron cone; however, the field strengths and gradients achievable will of necessity be reduced due to the very large apertures required to pass the neutron cone at this greater distance from the IP. 


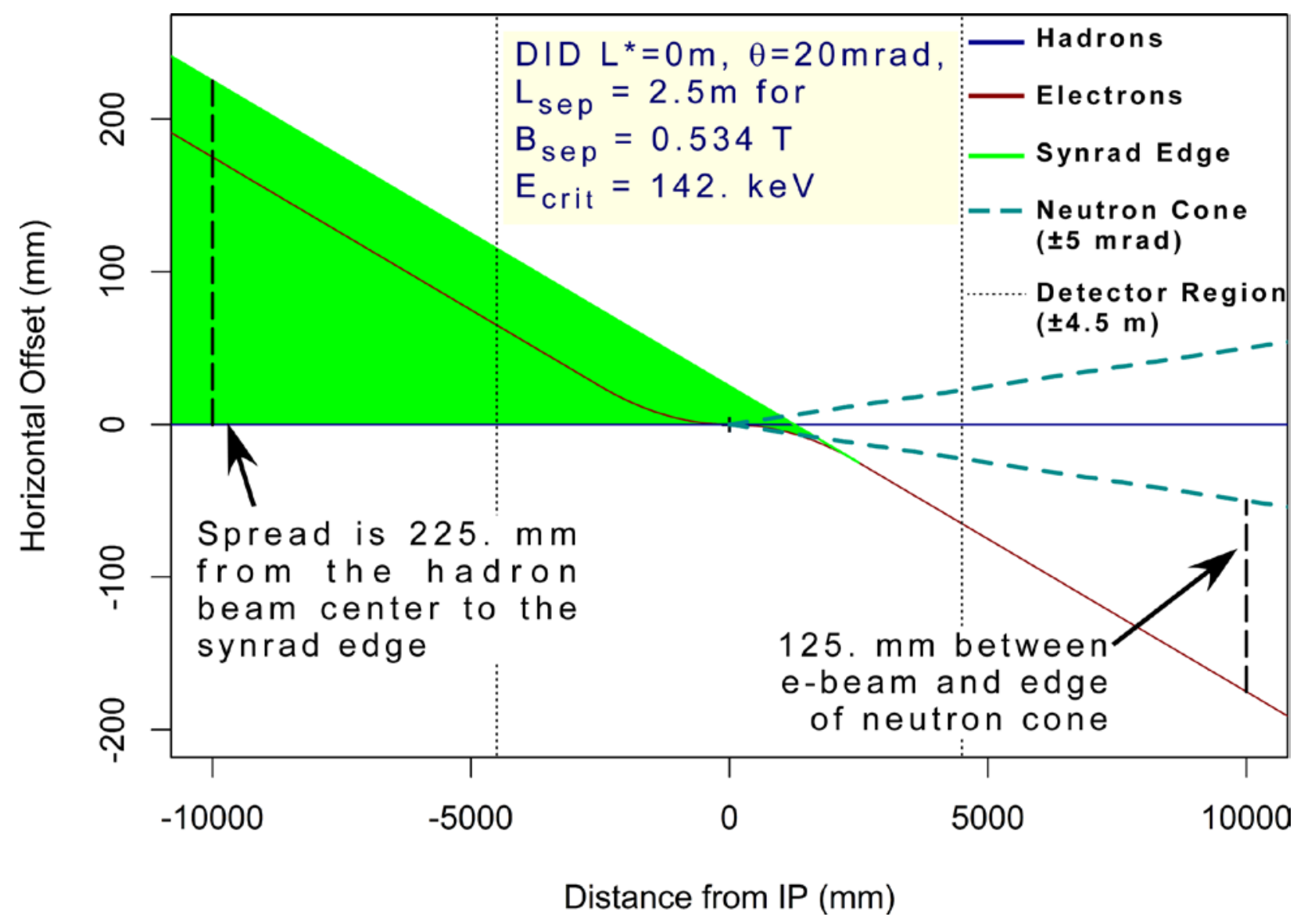

Figure 7: Scenario 3 with a more efficient modified eRHIC detector and DID coil geometry. Here the DID coil effective length is doubled while the common detector coil radius is halved so that the new geometry is more like the LHeC geometry. Unlike the LHeC geometry we do not use additional warm separation dipole sections outside the detector in order to preserve some forward/rear acceptance. With 20 mrad e-beam deflection angle there is now adequate space at $10 \mathrm{~m}$ in the forward direction for a superconducting coil structure to start focusing the hadron beam and separating the forward charged particles from the neutron cone; however, the field strengths and gradients achievable will of necessity be reduced due to the very large apertures required to pass the neutron cone further from the IP. There is also an issue on the rear side that it is very difficult to protect against synrad backscatter albedo that can directly shine on sensitive central detector tracker components. Since anti-backscatter masks cannot extend into the synrad fan without themselves becoming sources of background, the main mitigation measure is to further increase the radial extent of the central beam pipe and accept some further loss of physics.

Next we address an issue that with the eA IR geometry that is easily overlooked, e.g. requiring a large separation bend angle to clear the 5 mrad neutron cone also implies that on the rear side the synrad fan is very wide and quite challenging to deal with when using superconducting IR magnets. In this way the forward acceptance requirements also directly impact the rear side IR design configuration. In general there will always be some magnet structure on the rear side between the high field region for the incoming hadron beam and the reduced field region for the outgoing electrons and a synrad absorber will be needed to protect this material. However, striking such an absorber with synrad having critical energies $100 \mathrm{keV}$ and above generates abundant albedo backscatter photons that can become a potent background source by streaming back and hitting sensitive detector elements. Parenthetically we note that if the critical energy is pushed yet higher (e.g. greater bend angles and/or shorter magnets), then albedo neutrons produced via nuclear giant resonance interactions can also become a concern for the detector. The two means available to deal with such a backscatter background 
are increasing the distance from the background source to the detector and providing intermediate antibackscatter masks and shielding. Some of the principles and tradeoffs for designing an effective antibackscatter scheme are illustrated schematically in Figure 8.

Synrad Geometry with a Single Central Absorber

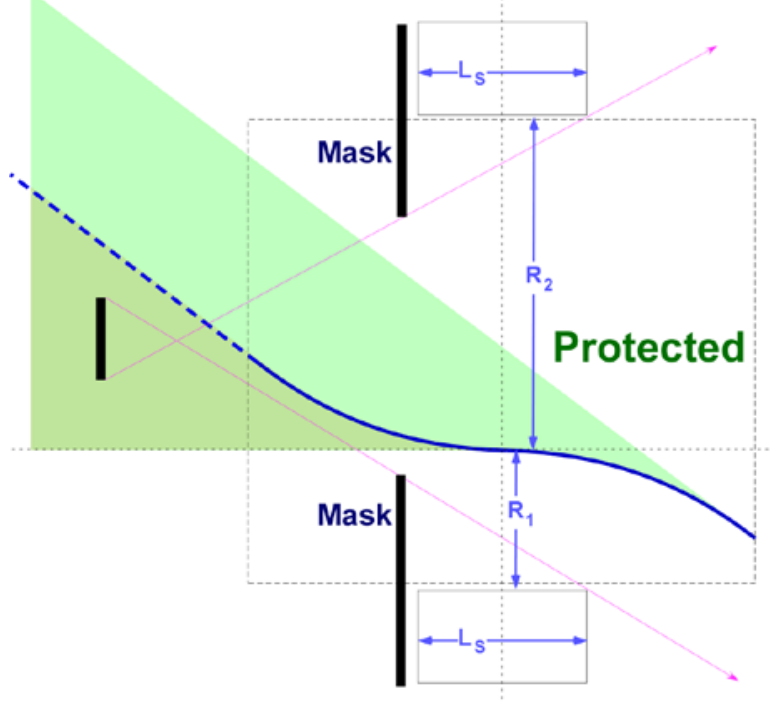

$L_{s}=$ Length of sensitive region to be protected

$\mathbf{R}_{\mathbf{1}}=$ Distance to synrad outer sensitive region

$\mathbf{R}_{\mathbf{2}}=$ Distance to synrad inner sensitive region

\section{Synrad Geometry with Large Angle Absorber Added}
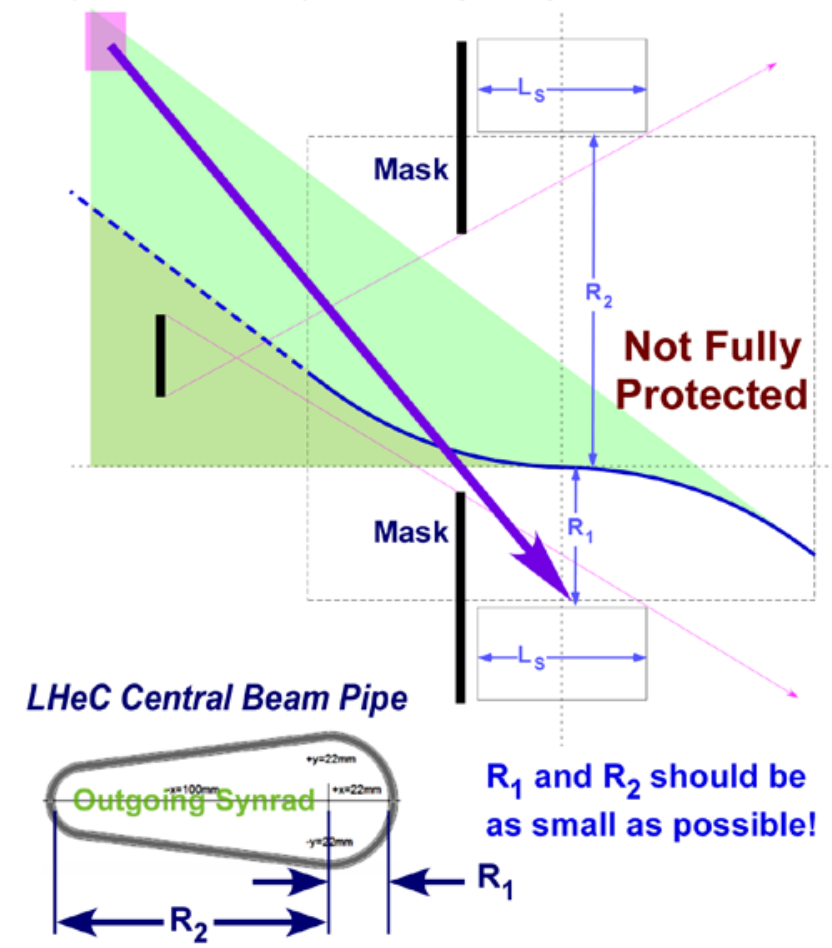

Figure 8: Schematic illustration for issues associated with protecting central detector region against synrad albedo backscatter. For the s-bend beam separation geometry the synrad fan folds back on itself and the outer edge extends constant distance from the exiting e-beam. On the left we see an example where there is a narrow absorber at a small angle that is used to protect the superconducting IR magnets and the rest of the fan passes along with the exiting e-beam out through an extended cutout region. In this case we see that it is possible to protect sensitive central detector components against line-of-sight backscatter from this absorber via masks positioned outside the main synrad fan. The insert shows the LHeC central beam pipe design that is extended on one side due to the outgoing synrad band but is as close as possible to the collision axis on the other side in order to make the best possible physics measurements. But on the left side we see what happens if a portion of the outer edge of the synrad fan hits something. Now there are rays that can come back at a steep angle and miss the anti-backscatter masks. Since we assume that the original masks are already placed as close as possible so as not themselves to be a source of beam loss and background, then we must either make R1 larger or the sensitive region shorter, i.e. lose physics. Note passing the full synrad fan far from the IP was possible for HERA-II because warm, normal conducting, magnets were used where it was possible to split the coils vertically to make a clear path for synrad; for the field strengths assumed in the eRHIC design these magnets are superconducting and it becomes impractical to extend any such cutout regions far from the IP (especially if we want to shield the e-beam from significant stray fields). It is sometimes suggested that anti-backscatter masks could be placed above/below the exiting synrad fan to partially mitigate this background; however, any such masks that significantly reduce the total solid angle for backscatter also reduce particle physics acceptance in the rear direction.

For the s-bend beam separation geometry depicted in Figure 8, the synrad fan folds back on itself and the outer synrad fan edge extends constant distance from the exiting e-beam. On the left we see an example where there is a narrow absorber at a small angle that is used to protect the superconducting IR magnets and the rest of the fan passes along with the exiting e-beam out through an 
extended cutout region. In this case it is possible to protect sensitive central detector components against line-of-sight backscatter from this absorber via masks positioned outside the main synrad fan and circulating beam envelopes. Note though that for such a masking solution to exist it is necessary to provide some significant distance between the end of the synrad source, the separation dipole, and the absorber; it is a mistake to place a synrad absorber immediately after the separation bend because then the masks cannot prevent line of sight backscatter from hitting central detector components unless the detector element are retracted far from the colliding beams which then has a major impact on physics. In general the experiments want to place tracking detectors as close as possible to the colliding beams. For example an insert on Figure 7 shows how the LHeC central beam pipe design is extended on one side just enough to pass the outgoing synrad band but is as close as possible to the collision axis on the other side in order to make the best possible physics measurements; the LHeC experiment has had to increase the beam pipe on the side with the synrad fan but they at least look to get some tracking elements close to the beam on the opposite side.

But now on the left side of Figure 8 we consider what happens if a portion of the outer edge of the synrad fan hits something. Now in spite of the anti-backscatter masks to protect against backscatter from the septum protection absorber, there are rays that can come back at a steep angle and miss these masks. Assuming that the original masks are already placed as close as possible so as not themselves to be a source of beam loss and background, then we must either make the close in beam pipe dimension, $\mathrm{R} 1$, larger or the sensitive region length shorter, i.e. lose physics. Eventually all synrad has to hit somewhere; so if we cannot afford to touch any of the outer edge of the synrad band close to the detector, then the only hope for attenuating the flux of backscatter albedo background is to pass the bulk of the synrad fan far enough away from the experiment that the solid angle for backscatter to see the detector is sufficiently small.

This strategy, passing the full synrad fan far from the IP, was possible for HERA-II because warm, normal conducting, magnets could be used where it was possible to split the coils vertically to make a clear path for synrad; for the field strengths assumed in the eRHIC design, these magnets will be superconducting and it becomes impractical to extend any such cutout regions very far from the IP (especially if we want to shield the e-beam from significant stray fields). It is sometimes suggested that anti-backscatter masks could be placed above/below the synrad fan inside the detector to partially mitigate this background; however, any masks that significantly reduce the total solid angle for backscatter will certainly cut into particle physics acceptance in the rear direction.

It has also been suggested that, in the spirit of a staged upgrade approach, one might consider using such a DID separation dipole scheme to do an initial program of eA physics, where there is interesting physics that can be done with lower luminosity, and then later upgrade the scheme to use a crossing angle to more aggressively push magnets closer to the IP to reach an ultimate luminosity. Unfortunately the IR magnets for a given separation scheme, if they are well optimized to give the best performance, end up being carefully tailored to the specific geometry they were optimized for and are generally not reusable. More fundamentally going from one scheme to another is more than just a matter of building an extra DID coil into the detector and later turning it off when we want to go over to a crossing angle solution. As we have seen, for a DID crossing angle scheme to be compatible with the main requirements to do eA physics, the experimentalists must adopt significant compromises that strongly impact the detector solenoid geometry and available detector subsystems. It would seem desirable not to force DID constraints at the outset but rather to leave the experimentalist the freedom to look to make the most suitable technology choices and tradeoffs for covering both an initial eA program as well as the ultimate high-luminosity physics. Otherwise there is a strong possibility that in addition to accelerator upgrades much of the detector itself would have to be rebuilt as part of an overall upgrade. Also inherent in such a plan is that after performing such an upgrade it is then difficult to go back the other direction and make any new eA physics measurements if there were to be future physics surprises that might warrant revisiting some eA kinematic range. Finally it is by no means clear 
that overlapping the detector solenoidal fields with strong dipole components can be done without having strong implications for spin matching of the hadron beam. Spin matching and polarization is a subject which we do not want to dwell on further except to observe that having a polarized hadron beam is a new regime for eRHIC to explore that was just not possible to do at HERA-II.

\section{Impact of this IR design on the detector design and the EIC physics}

\subsection{Impact on Particle Identification at rapidities $|\eta|>1$}

For the EIC Physics it is extremely critical to separate pions, kaons and protons over a wide range in momentum and rapidity [2]. Figure 9 shows the momentum distribution of negatively charged pions (blue), kaons (cyan) and antiprotons (violet) for different pseudo-rapidity bins. For $\eta>1$ the momenta vary between $0.1 \mathrm{GeV}$ to $100 \mathrm{GeV}$ at a center-of-mass energy of $122 \mathrm{GeV}$.
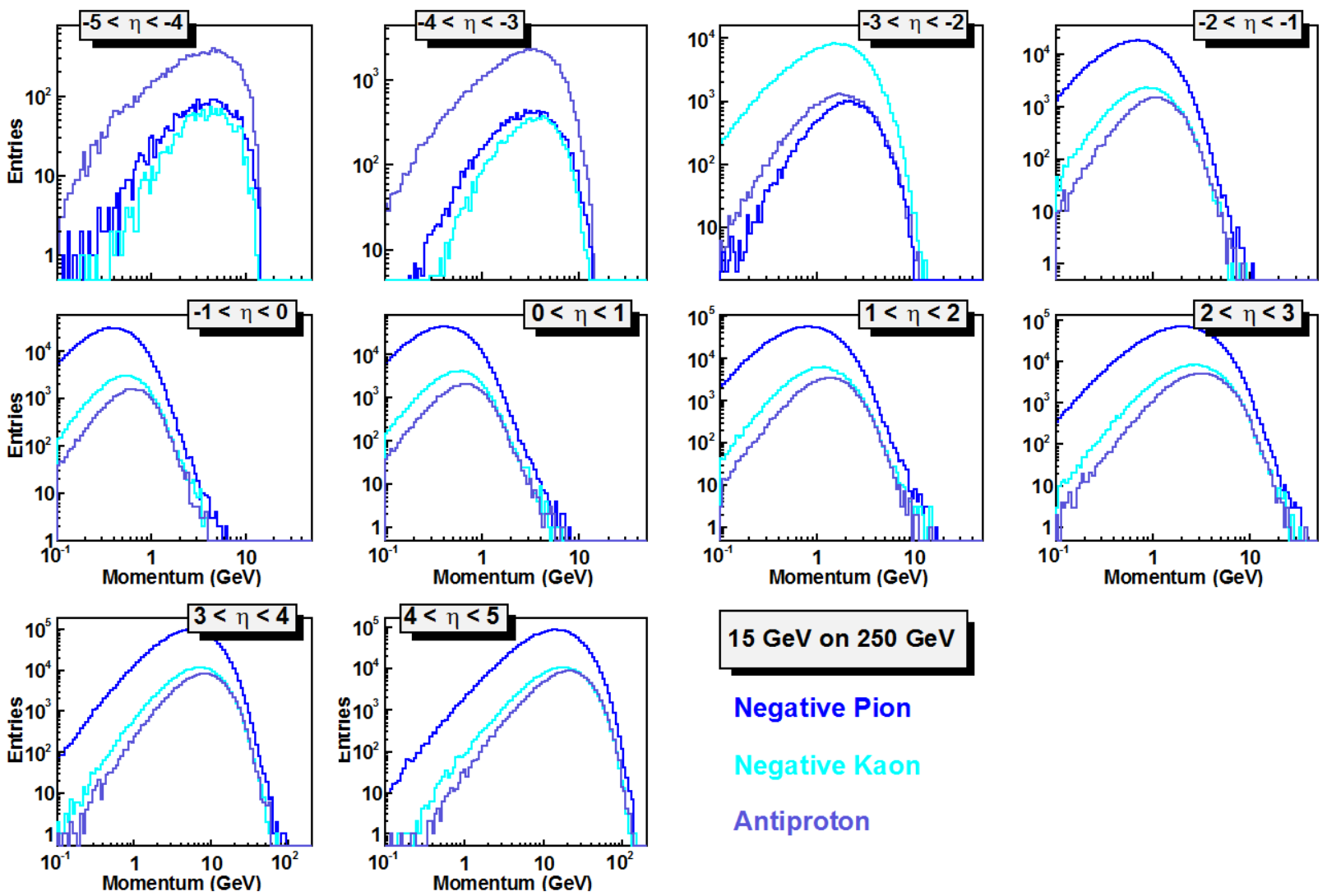

$15 \mathrm{GeV}$ on $250 \mathrm{GeV}$

Negative Pion

Negative Kaon

Antiproton

Figure 9: The momentum distribution negatively charged pions (blue), kaons (cyan) and antiprotons (violet) for different pseudo-rapidity bins in the laboratory frame for beam energies of $15 \mathrm{GeV}$ on $250 \mathrm{GeV}$. No kinematic cuts have been applied.

As EIC is planning to run at different center-of-mass energies the question arises how this momentum distributions change with center of mass energy,

Figure 10 shows the momentum versus pseudo-rapidity distributions in the laboratory frame for pions originating from semi-inclusive reactions for different lepton and proton beam energy combinations. For increasing hadron beam energies at fixed lepton beam energy the pseudo-rapidity distribution remains the same but the maximum hadron momentum increases at fixed pseudo-rapidity. The kinematic distributions for kaons and protons/anti-protons are essentially identical to those of the 
pions. The distributions for semi-inclusive events in electron-nucleus collisions may be slightly altered due to nuclear modification effects, but the global features will remain.

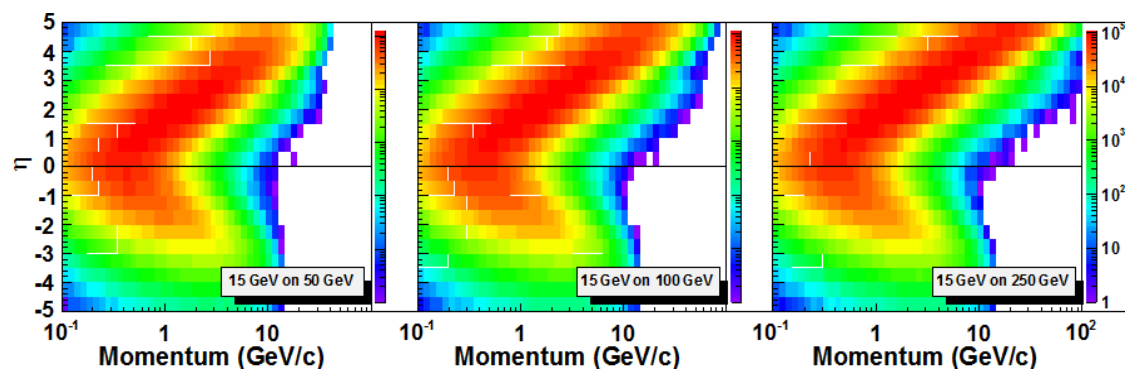

Figure 10: Momentum vs. pseudo-rapidity in the laboratory frame for pions from non-exclusive reactions at different center-of-mass energies. The following cuts have been applied: Q2 > 1 $\mathrm{GeV}^{2} 0.01<\mathrm{y}<0.95$.

Separating pions, kaons and protons over this wide momentum range needs special particle identification detectors. In the following the world wide available different technologies and the impact of having a separation dipole integrated into the detector are discussed.

1. Particle ID through $\mathrm{dE} / \mathrm{dx}$ in the TPC. This technology covers the low momentum range up to $\sim 0.5$ $\mathrm{GeV}$. But as the integrated dipole makes the use of a TPC basically impossible as discussed earlier, this technology is not available.

2. Another technology used for low momentum particles is time-of-flight (ToF), which requires to place a detector at a distance as large as possible from the interaction region, as further away the better the separation for pions, kaons and protons as function of momentum will be. The current design allows for a flight path of $2.5 \mathrm{~m}$ to $3 \mathrm{~m}$, which would depend on the intrinsic resolution. For a 10ps intrinsic detector resolution and a flight path of $3 \mathrm{~m}$ a pion-kaon separation can be reached up to $4 \mathrm{GeV}$. This technology does not provide the momentum coverage needed for the physics. As such ToF is a technology, which needs to be combined with other detectors to cover the full hadron momentum range. There is one other caveat to be made, the ToF technology, which fulfills the requirement to be magnetic field insensitive and covers the rapidity $1<\eta<4$ might be prohibitively expensive.

3. The most promising PID technology to separate pions, kaons and protons over the entire momentum range are dual radiator ring imaging Cerenkov detectors (RICH). These would involve a radiator with a high refractive index as AEROGEL covering the momentum region from 0.5 to 20 $\mathrm{GeV}$ and a gas radiator with a low refractive index as $\mathrm{C}_{4} \mathrm{~F}_{10}$ covering the momentum region from 5 $\mathrm{GeV}$ to $60 \mathrm{GeV}$. If the entire RICH detector will have to sit in the dipole field there are a couple of consequences. To detect the Cerenkov photons only a magnetic field insensitive technology is possible. One possible technology would be a photon detector based on GEM, but unfortunately due to the sensitivity of GEMs to only low wavelength photons $<150 \mathrm{~nm}$, this would be incompatible with having Aerogel as radiator as its Cerenkov photons are in the 400nm region. Unfortunately having a replacement for Aerogel is not an easy task as there are no other radiators currently available having the same refractive index. This would leave a photon detector option only SiPMs, an option currently investigated by the BELLE collaboration. Gas Cerenkov radiators in general span a length of $1 \mathrm{~m}$, which if the RICH sits in a magnetic field would mean, the cone of the emitted Cerenkov photons gets smeared out, because the track corresponding to the charge particle emitting the Cerenkov photons is bent going through the radiator. This will lead to a significant loss in pion, kaon, proton separation. 
In summary, a dipole integrated in the detector has significant impact on the particle identification capabilities by excluding available technologies or impacting the performance of specific particle detectors.

\subsection{Impact on tracker performance}

The influence of the detector-integrated dipole installation on the model detector tracker was investigated as well. Magnetic field map of such a dipole was imported in EicRoot software framework and basic momentum resolution studies performed. The tracker momentum resolution in the "default" configuration with the $~ 3 T$ solenoid field only is shown in Figure 11 for different hadron momenta as a function of pseudo-rapidity. Due to the solenoid field symmetry this resolution does not depend on the azimuthal angle of secondary particle tracks.

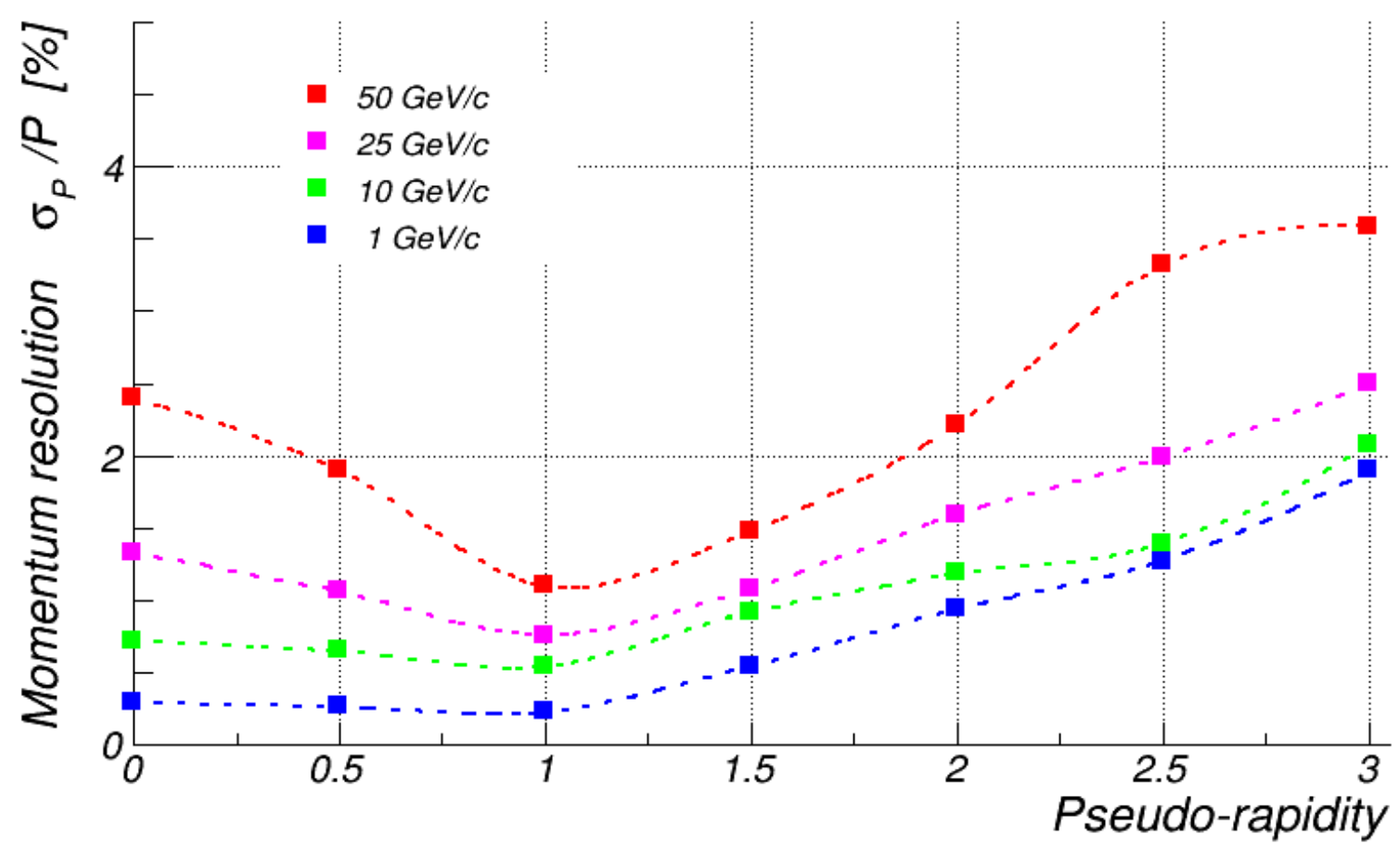

Figure 11: Tracker momentum resolution in [\%] for pions of four different momenta as a function of pseudorapidity. Solenoid field only. Anticipated model detector tracker provides resolution below $\sim 3 \%$ in almost the whole kinematic range of interest for physics.

The situation changes when a strong dipole field with a different symmetry type is added. Figure 12 shows for example the momentum resolution for $10 \mathrm{GeV} / \mathrm{c}$ pions at different values of azimuthal angle. One can clearly see, that not only does the detector lose the original azimuthal symmetry, desirable for suppressing acceptance-related systematics in the data analysis, but also the resolution becomes very poor at certain azimuthal angles around pseudo-rapidity value of $\sim 2.5$ due to the approximate cancellation of solenoid and dipole fields. Similar behavior is observed for other momentum values of secondary charged particles as well. 


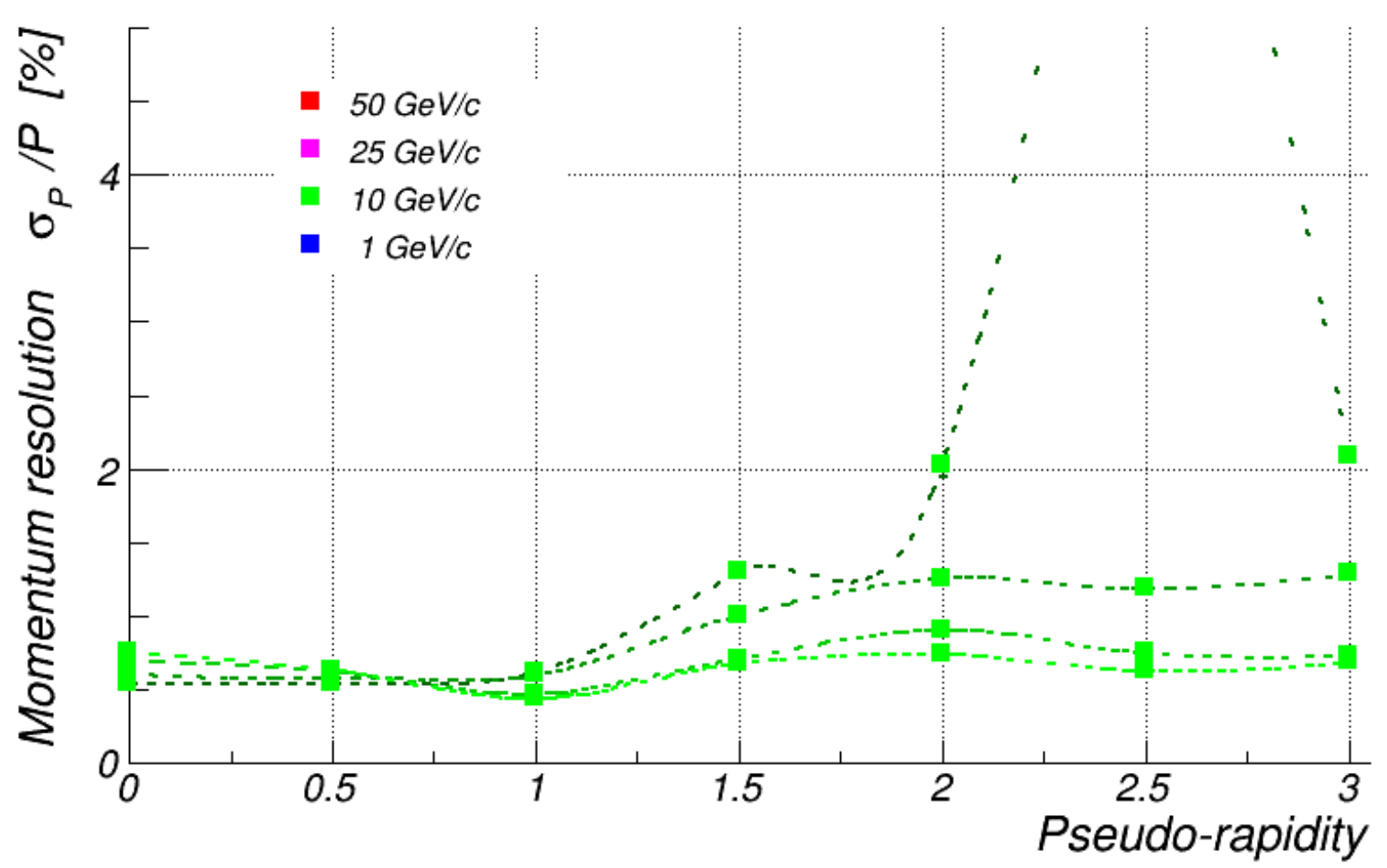

Figure 12: Tracker momentum resolution in [\%] for $10 \mathrm{GeV} / \mathrm{c}$ pions at four different values of azimuthal production angle as a function of pseudo-rapidity. Superposition of solenoid and dipole fields. The different curves represent azimuthal angles $-90^{\circ},-30^{\circ},+30^{\circ}$ and $+90^{\circ}$ from bottom to top, with $0^{\circ}$ being in the horizontal direction.

Figure 13 illustrates this undesirable effect in a different way. The integral $\left|B_{t}\right| d l$ of transverseto-track magnetic field component, essential for the momentum resolution, is shown for the "nominal" case of the solenoid field only (left) and the case when the detector-integrated dipole field is added on top of it (right). The right panel clearly shows, that the tracker loses azimuthal symmetry and there are spots in acceptance with very small field integral (and consequently, very poor momentum resolution).
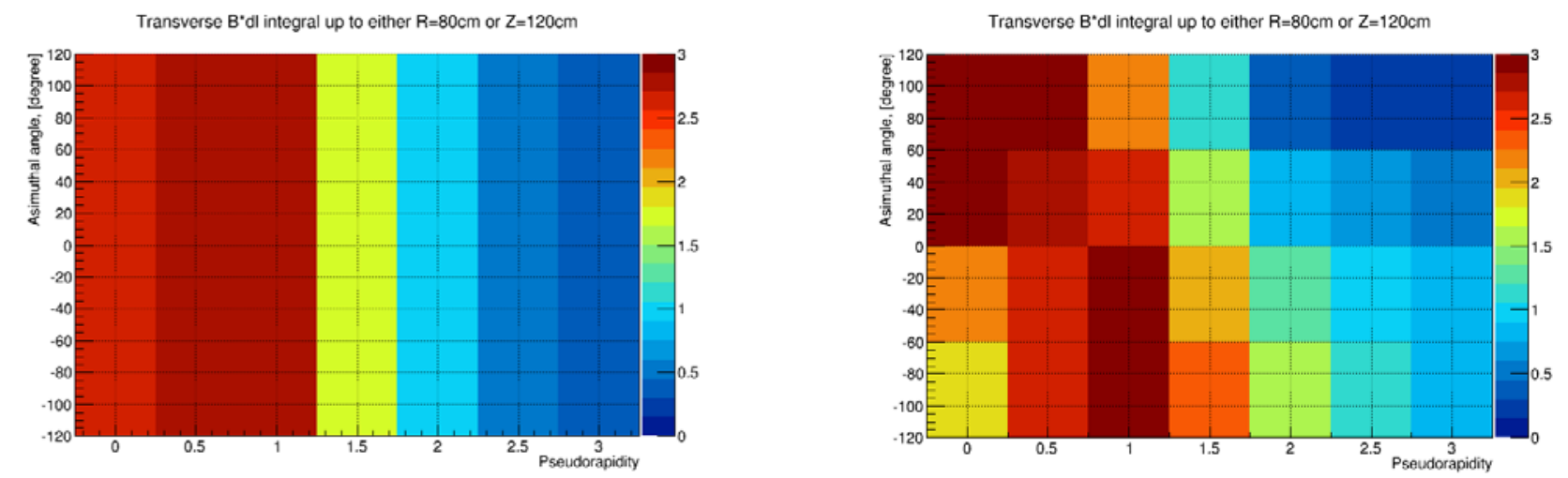

Figure 13: Transverse-to-track magnetic field component integral over the particle trajectory as a function of pseudo-rapidity and azimuthal angle. Left panel: solenoid field only. Right panel: superposition of solenoid and dipole fields.

Even worse, it turns out, that the operation of a TPC is basically impossible in case of the presence of a dipole field. This is clearly seen in Figure 14, where the magnetic field component, transverse to the direction of the TPC electric field (which is naturally aligned with the beam line) is shown for the whole anticipated TPC gas volume (up to $+/-80-100 \mathrm{~cm}$ in the beam direction and up to 
60-80 $\mathrm{cm}$ in the radial direction away from the nominal IP). The left plot shows, that in case of a pure solenoid field at least for a smaller size TPC the transverse magnetic field component can be maintained well below $\sim 1 \mathrm{kGs}$ in most part of the gas volume even for this very preliminary compact solenoid design. In case when the dipole field is added (right panel) the strong inhomogeneous intrinsically transverse magnetic field component is present in a large fraction of the TPC gas volume, reaching values of up to $\sim 1 \mathrm{~T}$ and more at some azimuthal angles, and it can clearly not be removed by any possible magnet layout optimization. This fact alone requires a complete tracker re-design with the likely degradation of either the basic detector properties essential for the DIS experiment (increase in radiation length thickness, etc) or performance (lower spatial resolution, no PID via dE/dx, etc) or both.
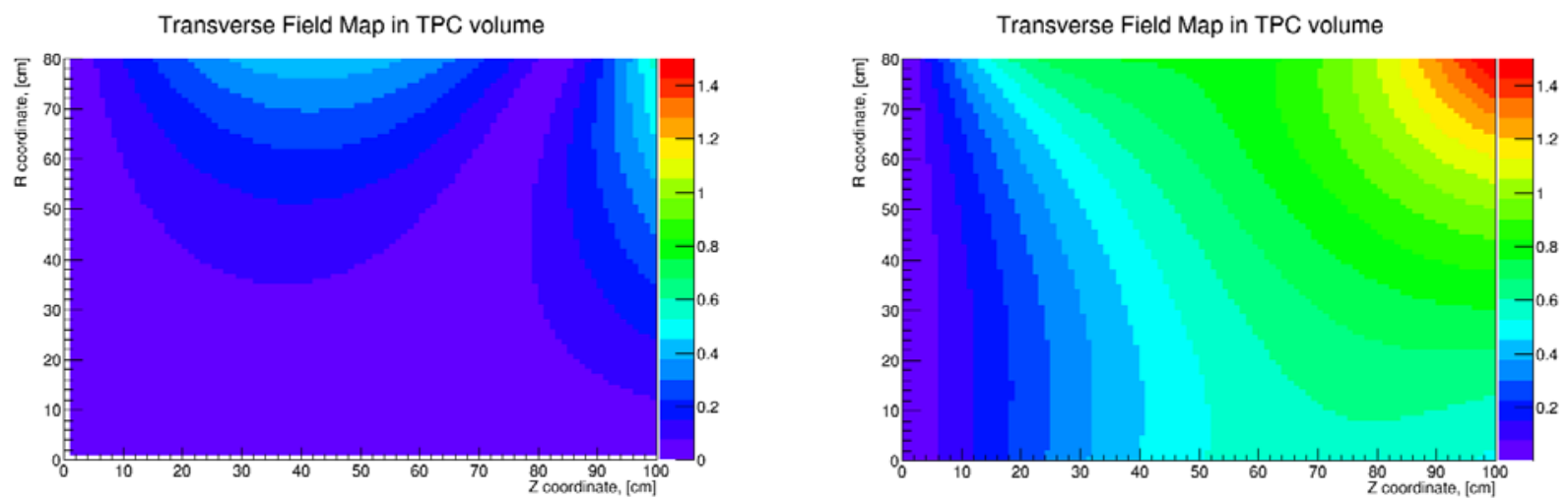

Figure 14: Transverse-to-beam-line magnetic field component in the anticipated TPC installation volume. Left panel: solenoid field only. Right panel: superposition of solenoid and dipole fields at azimuthal angle of 90 degrees (extreme case).

\subsection{Impact on auxiliary detectors}

Any version of the scenarios using a dipole field to separate the beams will have impact on the performance of the auxiliary detectors described shortly in the following.

1. The acceptance of the Roman Pot detectors for forward going protons will be modestly affected for scattered protons with significantly lower momentum as the beam energy.

2. The "low-Q $\mathrm{Q}^{2}$-tagger", the detector in the outgoing electron direction to detect the scattered leptons with small scattering angles, will become basically impossible. The current IR design foresees to have this detector located $15 \mathrm{~m}$ away from the IP. The load of the synchrotron radiation hitting this detector will make any precision energy measurement of the scattered electron basically impossible. In addition it will be close to impossible to find any electromagnetic calorimeter material, which provides high energy-resolution and is radiation hard enough to stand the radiation load.

3. The luminosity detector currently planned at $35 \mathrm{~m}$ in the outgoing electron beam direction from the IR will also very much affected from the synchrotron radiation. The luminosity in an EIC is measured through bremsstrahlung, the design already integrated a pair spectrometer as a calorimeter under zero degree would be impacted also in the std. eRHIC linac-ring design from synchrotron radiation, which could influence a high precision luminosity measurement, but the wide synchrotron radiation fans seen in the above presented IR scenarios based on a separation dipole might yield even problems to the pair spectrometer based luminosity measurement. This needs to be carefully modeled and shielding needs to be integrated into the machine design to ensure a high precision ( $<1 \%$ systematic uncertainty) luminosity measurement. 


\subsection{Experimental Impact Summary}

In the following the impact on the EIC detector design as well as on the physics is summarized.

1. A standard DIS detector design is impossible with scenario 3, the impacts on realizing the physics program described in [2].

2. The DID design impacts the possible technology choices for the main tracker and the particle ID. To have not all technologies available increases the risk of the detector to reach the performance needed for the physics program. As example not having PID at small momenta is exactly the kinematic regime where saturation is studied. In general compromising on PID impacts all SIDIS measurements planned for ep and eA physics at an EIC. To not have the possibility to have a TPC as central tracker will significantly compromise the material budget in the detector as any other solution providing comparable momentum resolutions will have more material. More material will automatically lead to a worse scattered lepton reconstruction and therefore the event kinematics.

3. The large size of the synchrotron radiation fan through the detector does impact how close the $\mu$ vertex tracker can go to the IR. The coverage will be asymmetric, as the fan has different dimensions in $\mathrm{y}$ than $\mathrm{x}$.

4. All low $\mathrm{Q}^{2}\left(\mathrm{Q}^{2}<0.1 \mathrm{GeV}^{2}\right)$ physics will become impossible having no low $\mathrm{Q}^{2}$ tagger. It is absolutely critical to make sure the synchrotron radiation does not prevent a luminosity measurement with $<1 \%$ systematic uncertainty.

To detail all the impact of a separation dipole on all the physics program of an EIC, a complete IR design needs to be finalized, a EIC detector needs to undergo a major redesign and then all the physics discussed in [2] needs to be reevaluated. This is beyond the scope of the currently available manpower.

[1] E.C. Aschenauer et al, IR Design Requirements, eRHIC wiki https://wiki.bnl.gov/eic/index.php/IR Design Requirements

[2] A. Accardi et al., The EIC White Paper “The Next QCD Frontier-Understanding the glue that binds us all” arXiv:1212.1701.

E.C. Aschenauer et al., eRHIC Design Study: An Electron-Ion Collider at BNL arXiv:1409.1633 